\title{
Relação entre divergência genética de acessos de berinjela e desempenho de seus híbridos.
}

\author{
DERLY J.H. SILVA ${ }^{1}$; CYRO P. COSTA²; VICENTE W.D. CASALI ${ }^{1}$, LUÍZ A.S. DIAS ${ }^{3}$; COSME D. \\ CRUZ ${ }^{4}$ \\ ${ }^{1}$ UFV - Dep ${ }^{\text {to }}$ de Fitotecnia, 36571-000 Viçosa, MG; ${ }^{2}$ USP - ESALQ - Dep ${ }^{\text {to }}$ Horticultura , C. Postal 9, 13418-900 Piracicaba, SP; \\ ${ }^{3}$ CEPLAC, atualmente UFV - Dep ${ }^{\text {to }}$ de Biologia Geral, 36571-000 Viçosa, MG; ${ }^{4}$ UFV - Dep ${ }^{\text {to }}$ de Biologia Geral, $36571-000$ Viçosa, MG.
}

\section{RESUMO}

A divergência genética $\left(\mathrm{D}^{2}\right)$ entre cinco acessos de berinjela foi estimada para avaliar a eficiência da diversidade na predição do desempenho dos híbridos respectivos. Os acessos PI 206472 e 'Campineira' foram os mais similares, enquanto 'Long Green' e PI 206472 foram os mais divergentes. A associação entre divergência genética dos genitores e desempenho dos híbridos foi analisada por três métodos, apresentando os seguintes resultados: 1) correlações de Pearson $\left(r_{p}\right)$ e Spearman $\left(r_{s}\right)$ : a divergência $\left(D^{2}\right)$, mostrou estar linearmente associada ao desempenho médio dos híbridos para o caráter número médio de frutos por planta $\left(\mathrm{r}_{\mathrm{p}}=0,71\right.$ e $\mathrm{r}_{\mathrm{S}}=0,636, \mathrm{P}$ $<0,05)$ e inversamente relacionada para produção de frutos $(\mathrm{g} / \mathrm{plan}-$ ta) $\left(r_{\mathrm{P}}=-0,687, \mathrm{P}<0,05\right)$; 2) separação em classes de cruzamentos divergentes: mostrou-se promissora, pois dois híbridos selecionados (PI 206472 x 'Florida Market' e 'Campineira' x 'Florida Market') ocuparam segunda e a quarta posição na classificação das médias, respectivamente; 3) método da classificação dupla: considerado eficiente, pois selecionou o cruzamento PI 206472 x 'Florida Market', que obteve a segunda posição na classificação das médias, superando os híbridos 'Nápoli' e 'Super F100', os quais são cultivados comercialmente.

Palavras-chave: Solanum melongena, berinjela, análise multivariada, diversidade genética, melhoramento.

\begin{abstract}
Relationship between genetic divergence and hybrid performance in eggplant.

The genetic divergence $\left(\mathrm{D}^{2}\right)$ among five accessions of eggplant was estimated to evaluate the efficiency of this divergence at predicting the performance of corresponding hybrids. The closest related accessions were PI 206472 and 'Campineira' whilst 'Long Green' and PI 206472 were the most divergent. The association between hybrid performance and genetic divergence of parents was analysed by three methods: 1) Pearson's $\left(r_{p}\right)$ and Spearman's $\left(r_{s}\right)$ correlation: genetic divergence $\left(\mathrm{D}^{2}\right)$ was found to be linearly related to hybrid performance of mean fruit number per plant $\left(\mathrm{r}_{\mathrm{p}}=0.71\right.$ and $\left.\mathrm{r}_{\mathrm{S}}=0.636, \mathrm{P}<0.05\right)$ and inversely related to fruit yield $\left.\left(r_{P}=-0.687, P<0.05\right) ; 2\right)$ Divergence of cross classes: This found to be successful because two selected hybrids (PI 206472 x 'Florida Market', 'Campineira' x 'Florida Market') reached $2^{\text {nd }}$ and $4^{\text {th }}$ place on the rank of means respectively; 3) Method of double rank: This was found to be efficient since it allowed the selection of the cross PI 206472 x 'Florida Market', which reached $2^{\text {nd }}$ place on the rank of means, overcoming the commercial hybrids 'Nápoli' and 'Super F100'.
\end{abstract}

Keywords : Solanum melongena, eggplant, multivariate analysis, genetic diversity, breeding.

\section{(Aceito para publicação em 22 de março de 1999)}

$\mathrm{O}$ mercado consumidor brasileiro exige cada vez mais produtos olerícolas com apresentação visual boa e uniforme, além de preço acessível. No caso da berinjela (S. melongena $\mathrm{L}$.) tais exigências levam à utilização de híbridos, em razão da uniformidade e da grande produção.

Para a obtenção de híbridos, o parâmetro de maior interesse é o desempenho médio dos híbridos (Cruz et al., 1994a). Os fatores genéticos que determinam este desempenho envolvem desvios de dominância dos caracteres, diferenças de freqüências gênicas entre genitores, efeitos gênicos de natureza aditiva, além dos efeitos epistáticos (Falconer \& Mackay, 1996).

Assim, Cruz et al. (1994b) concluíram que a hibridação de genitores divergentes possibilita maior efeito heterótico na progênie e maior probabilidade de recuperar genótipos superiores nas gerações segregantes. Atualmente diversos autores têm demonstrado a associação entre divergência genética entre genitores e desempenho dos respectivos híbridos (Amaral Júnior, 1996; Dias \& Kageyama, 1997; ManjarrezSandoval et al., 1997). Tal associação acontece, pois a divergência genética está relacionada à diferença de frequiência gênica entre genitores e, dessa forma, associada ao fenômeno da complementação gênica (Ghaderi, et al., , 1984), que por sua vez está ligada à heterose e à capacidade específica de combinação (Bernardo, 1992).

Entretanto, Arunachalam \& Bandyopadhyay (1984) estudando métodos de seleção de genitores, concluíram que existem limites para a associa- ção entre divergência genética, entre acessos, e desempenho dos híbridos e que, dentro desses limites, o número de cruzamentos com bom desempenho foi maior que fora destes. Neste sentido, Cruz et al. (1994b), sugeriram que os cruzamentos devem ser feitos entre genitores divergentes, mas que apresentem também desempenho superior em relação às características de interesse do melhorista. Segundo Lal \& Pathak (1974), trabalhando com berinjela, genitores com bom desempenho apresentam alta capacidade geral de combinação de combinação (Griffing, 1956), o que é indicativo de grande concentração de alelos favoráveis (Vencovsky, 1970).

Dessa forma, os objetivos do presente trabalho foram: (a) estimar a correlação existente entre divergência genética dos acessos e desempenho dos híbri- 
dos; e (b) avaliar a eficiência do procedimento de Arunachalam \& Bandyopadhyay (1984) e do método da classificação dupla na seleção de genitores. O método da classificação dupla consiste em classificar os genitores em relação aos caracteres avaliados e, posteriormente, selecionar, dentre os melhores, os pares mais divergentes para serem utilizados em cruzamentos.

\section{MATERIAL E MÉTODOS}

Utilizaram-se cinco acessos (Valois, 1996) de berinjela do Banco de Germoplasma de Hortaliças do Departamento de Genética da ESALQ/USP (1 - PI 206472; 2 - 'Long Green'; 3 'Campineira'; 4 - 'Florida Market' e 5 'E-22').

$\mathrm{O}$ experimento foi conduzido em Piracicaba-SP, no delineamento de blocos ao acaso, com três repetições e parcelas de oito plantas. Foram consideradas úteis as quatro plantas centrais. Os tratos culturais foram executados conforme Filgueira (1982).

De acordo com o método de Kempthorne \& Curnow (1961) para dialelo circulante, incluindo os genitores em duas combinações híbridas por genitor ( $\mathrm{s}=2$ ), foram analisados os genótipos: 1 ; $2 ; 3 ; 4 ; 5 ; 1 \times 3 ; 1 \times 4 ; 2 \times 4 ; 2$ x 5 e $3 \times 5$. As médias dos demais híbridos ( 1 x $2 ; 1 \times 5 ; 2 \times 3 ; 3$ x 4; 4 x 5), foram preditas conforme o método referido anteriormente, somando-se à estimativa da média geral as estimativas dos efeitos de capacidade geral de combinação dos respectivos genitores, cuja expressão é:

$$
\begin{aligned}
& \mathrm{Z}_{\mathrm{ij}}=\mu+g_{i}+g_{j} \\
& \text { em que }
\end{aligned}
$$

$\mathrm{Z}_{\mathrm{ij}}=$ média predita associada à combinação híbrida $\mathrm{ij}$ ( $\mathrm{i}^{1} \mathrm{j}$ );

$\mu=$ estimativa da média geral;

$g_{i}$ e $g_{j}=$ estimativas dos efeitos da capacidade geral de combinação, cujos valores encontram-se em Silva et al. (1999).

Foram feitas seis colheitas, tendo sido avaliados os seguintes caracteres, no ponto de colheita comercial:

- Comprimento médio de fruto (CMF), expresso em centímetros $(\mathrm{cm})$ - foi obtido medindo-se o comprimento médio de dez frutos, no sentido lon- gitudinal, por parcela, em cada colheita. Posteriormente, calculou-se o comprimento médio dos frutos a partir das estimativas dos comprimentos médios dos frutos obtidos em cada colheita. Este método foi utilizado para os caracteres DMF e PMF;

- Diâmetro médio de fruto (DMF), expresso em centímetros $(\mathrm{cm})$ - foi obtido medindo-se dez frutos transversalmente, na parte de maior diâmetro dos frutos, por colheita;

- Peso médio de fruto (PMF), expresso em gramas por fruto (g/fruto) foi obtido pela pesagem de dez frutos, por colheita, em balança com precisão de 1 (um) grama;

- Número médio de frutos por planta (NMFP) - somaram-se todos os frutos com padrão comercial, colhidos em cada parcela, e posteriormente dividiuse por quatro, número de plantas úteis por parcela;

- Produção total de frutos/planta (PROD): expresso em gramas por planta (g/planta) - foi obtida pela soma das pesagens de todos os frutos, com padrão comercial, colhidos em cada parcela, e posteriormente dividiu-se por quatro, número de plantas por parcela.

As análises de variâncias univariadas foram realizadas considerando-se como fontes de variações os efeitos de bloco, de acessos, de híbridos, do contraste acessos x híbridos e do erro experimental. Para se avaliar a confiabilidade dos valores fenotípicos médios em representar os valores genotípicos, foi estimado o coeficiente de determinação genotípica $\left(H^{2}\right)$, cuja expressão é:

$$
\begin{aligned}
& H^{2}=\left[\Phi_{g} / \sigma_{p}^{2}\right] \times 100 \\
& \text { em que }
\end{aligned}
$$

$\Phi_{g}=$ estimativa do componente quadrático associado aos efeitos genotípicos, para modelos fixos; e

$$
\sigma_{p}^{2}=\text { estimativa da variância }
$$
fenotípica.

A divergência genética entre acessos (Rao, 1952) foi estimada pela distância generalizada de Mahalanobis $\left(\mathrm{D}^{2}\right)$, que é definida como:

\section{$\mathrm{D}^{2}=d^{-1} d$}

em que $\underset{\sim}{d}$ é um vetor de diferenças entre médias dos acessos para todos os

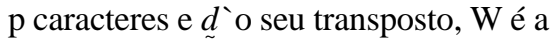

matriz p x p de variâncias e covariâncias residuais. Para delimitação dos grupos de similaridade, adotou-se a técnica conglomerativa de Tocher, conforme recomendado por Rao (1952).

Para testar a eficiência da associação entre divergência genética dos acessos e o desempenho médio dos seus híbridos foram utilizados três métodos:

1) estimação dos coeficientes de correlação de Pearson $\left(\mathrm{r}_{\mathrm{p}}\right)$ e de Spearman $\left(\mathrm{r}_{\mathrm{s}}\right.$ );

2) agrupamento dos acessos em classes de cruzamentos divergentes (CD); e

3) método da classificação dupla.

Método 1 - os coeficientes de correlação de Pearson e Spearman envolvem estimativas da Distâncias Generalizada de Mahalanobis $\left(\mathrm{D}^{2}\right)$, entre pares de genitores, e as médias dos respectivos híbridos, para os vários caracteres estudados (Steel et al., 1997). Este método é considerado eficiente se o coeficiente de correlação de $\mathrm{D}^{2}$, entre genitores, e as médias dos híbridos forem estatisticamente significativos, podendo portanto serem utilizados para selecionar genitores.

Método 2 - conforme Arunachalam \& Bandyopadhyay (1984), foram calculados a média (m) e o desvio-padrão (s) dos valores de divergência $\left(\mathrm{D}^{2}\right)$ entre acessos. As classes de divergência (CD) foram definidas como se segue:

$$
\begin{aligned}
& \text { CD1: } \mathrm{D}^{23} \mathrm{~m}+\mathrm{s} ; \\
& \text { CD2: } \mathrm{m}+\mathrm{s}>\mathrm{D}^{2} 3 \mathrm{~m} \\
& \text { CD3: } \mathrm{m}>\mathrm{D}^{2}{ }^{3} \mathrm{~m}-\mathrm{s} \text {; e } \\
& \text { CD4: } \mathrm{D}^{2}<\mathrm{m}-\mathrm{s} \text {. }
\end{aligned}
$$

Os cruzamentos foram distribuídos nas diferentes classes, segundo a divergência entre os genitores. Neste método, a classificação é considerada satisfatória se as classes CD2 e CD3, contiverem os cruzamentos com melhores desempenhos.

Método 3 - método da classificação dupla, que consiste em:

a) Fazer a classificação das estimativas das médias dos acessos para todos os caracteres analisados. Isto consiste em classificar os acessos, em relação a cada um dos caracteres, em ordem favorável ao melhoramento e somar os valores dos postos de classificação dos mesmos. Para o presente trabalho, buscou-se aumentar o valor dos caracteres estudados. Os melhores acessos foram 
classificados nas primeiras posições, com relação a cada caráter e, dessa forma, apresentaram as menores somas de postos na classificação.

b) Selecionar os acessos com menores somas de postos, descartando-se aqueles que apresentarem somas de postos de classificações com valores altos, em relação aos demais.

c) A partir do acesso com menor soma de postos de classificação, fazer a classificação com base na estatística $\mathrm{D}^{2}$ de Mahalanobis, buscando-se o acesso que apresenta a maior divergência genética. Assim, seleciona-se o cruzamento a ser feito. A eficiência deste método é avaliada em função da posição do híbrido, obtido no cruzamento selecionado, em relação aos demais.

Para efeito de comparação, foram incluídos os híbridos 'Nápoli' e 'Super F100'. Em todas as análises foi utilizado o programa GENES (Cruz, 1997).

\section{RESULTADOS E DISCUSSÃO}

Observaram-se diferenças significativas entre acessos $(\mathrm{P}<0,01)$, para todos os caracteres analisados (Quadro 1). Esta constatação indica facilidade na discriminação das melhores combinações híbridas, feitas com base em divergência genética. Quanto aos híbridos, somente as diferenças relacionadas ao caráter PROD não foram significativas; em razão disso, este caráter não foi con- siderado no cálculo da soma de postos de classificação, para híbridos (Quadro 2). Com relação aos caracteres analisados, detectaram-se boa precisão experimental $\left(\mathrm{CV}_{\mathrm{e}}<16 \%\right)$ e coeficiente de determinação genotípica $\left(H^{2}\right)$ acima de $80 \%$, indicando alta confiabilidade dos valores fenotípicos em representar os valores genotípicos.

Analisando os Quadros (2 e 3), em relação à distância genética $\left(\mathrm{D}^{2}\right)$, observaram que os acessos 1 (PI 206472) e 3 ('Campineira') foram os mais similares $\left(\mathrm{D}^{2}=4,578\right)$ e PI 206472 e 'Long Green' os mais divergentes $\left(\mathrm{D}^{2}=616,378\right)$. A identificação de pares de acessos com alta divergência tem sido objeto de muitos programas de melhoramento relacionados à obtenção de híbridos, visando maximizar a heterose e aumentar a probabilidade de ocorrência de segregantes superiores em gerações avançadas (Cruz et al., 1994b), bem como garantir base genética ampla para o início do programa de melhoramento (Hallauer \& Miranda Filho, 1988).

Em relação à associação entre divergência genética dos genitores e desempenho dos híbridos, método 1, observaram-se que (Quadro 2), para o caráter número médio de frutos por planta (NMFP), as correlações de Pearson e de Spearman, apresentaram associações significativamente positivas $(\mathrm{P}<0,05)$. Assim, à medida que aumenta-se o valor de $\mathrm{D}^{2}$ entre acessos, aumenta-se tam- bém o número de frutos do híbrido resultante do cruzamento dos mesmos.

Quanto aos caracteres CMF, DMF e PMF (Quadro 2) as correlações entre D 2 e o desempenho dos híbridos, foram consideradas estatisticamente nulas. Segundo Ghaderi et al. (1984), correlações nulas podem ser explicadas pela existência de dominância bidirecional, em função do cancelamento dos desvios de dominância positivos pelos negativos, e vice-versa, entre os genitores. Resultados semelhantes foram obtidos por Silva et al. (1999).

Foi observada correlação negativa (Quadro 2) entre a divergência entre acessos $\left(\mathrm{D}^{2}\right)$ e o desempenho dos híbridos para o caráter PROD $\left(r_{P}=-0,687\right.$, $\mathrm{P}<0,05)$ demonstrando associação linear inversa. Dessa forma, à medida que se aumenta a estimativa de $\mathrm{D}^{2}$ entre genitores, tende-se a reduzir a produção de frutos do híbrido resultante do cruzamento dos mesmos. Esse fato pode ser observado em relação aos genitores 1 e 2, os mais divergentes (Quadro 2), cujo híbrido apresentou estimativa menor para o caráter PROD que o híbrido obtido do cruzamento dos genitores 1 e 3, os mais similares (Quadro 2).

Estimativas de correlação negativa entre divergência dos genitores e desempenho dos híbridos foram observadas por Ghaderi et al. (1984) para o caráter número de dias para o florescimento em feijão (Phaseolus vulgaris L.) e fava

Quadro 1 - Resumo das análises de variância de cinco caracteres relativos à produção de frutos em berinjela. Piracicaba, USP-ESALQ, 1996.

\begin{tabular}{|c|c|c|c|c|c|c|}
\hline \multirow{2}{*}{ FV } & \multirow{2}{*}{ GL } & \multicolumn{5}{|c|}{ Q.M. } \\
\hline & & $\mathrm{CMF}^{1}$ & $\mathrm{DMF}^{2}$ & $\mathrm{PMF}^{3}$ & NMFP $^{4}$ & PROD $^{5}$ \\
\hline Blocos & 2 & 1,288 & 0,030 & 269,160 & 9,680 & 177565,820 \\
\hline Tratamentos & 9 & $9,714^{* *}$ & $7,490 * *$ & $22734,010 * *$ & $178,800 * *$ & 883341,420 ** \\
\hline Acessos $(A)$ & 4 & $15,205^{* *}$ & $11,653 * *$ & 33475,704 ** & 327,980 ** & 1274289,100 ** \\
\hline Híbridos (H) & 4 & $5,206 * *$ & $5,199 * *$ & 17301,850 ** & 65,110 ** & 436657,870 \\
\hline A vs $\mathrm{H}$ & 1 & $5,780 * *$ & $0,002 n s$ & $1495,873 *$ & $36,840 *$ & 1106284,900 ** \\
\hline Resíduo & 18 & 0,549 & 0,093 & 300,990 & 6,410 & 163326,015 \\
\hline Média & & 13,910 & 6,254 & 190,950 & 16,280 & 2529,060 \\
\hline C.V.(\%) & & 5,320 & 4,880 & 9,090 & 8,59 & 15,980 \\
\hline $\mathrm{H}^{2}(\%)$ & & 94,35 & 98,75 & 98,67 & 96,41 & 81,51 \\
\hline
\end{tabular}

**; *; $\mathrm{P}<0,01$ e $\mathrm{P}<0,05$ respectivamente.

${ }^{1} \mathrm{CMF}$ : comprimento médio de fruto; ${ }^{2} \mathrm{DMF}$ : diâmetro médio de fruto; ${ }^{3} \mathrm{PMF}$ : peso médio de fruto; ${ }^{4} \mathrm{NMFP}: \mathrm{n}^{\mathrm{o}}$ médio de frutos por planta; ${ }^{5}$ PROD: produção de frutos. 
Quadro 2 - Estimativas da divergência genética entre pares de acessos $\left(\mathrm{D}^{2}\right)$, das médias dos híbridos referentes a cinco caracteres relacionados à produção de frutos, das correlações de Pearson $\left(\mathrm{r}_{\mathrm{p}}\right)$ e Spearman $\left(\mathrm{r}_{\mathrm{S}}\right)$ entre $\mathrm{D}^{2}$ e as médias dos híbridos, e soma de postos na classificação das médias dos híbridos, para estes caracteres. Piracicaba, USP-ESALQ, 1996.

\begin{tabular}{|c|c|c|c|c|c|c|c|}
\hline Híbridos $^{a}$ & $\mathrm{D}^{2}$ & $\mathrm{CMF}^{\mathrm{b}}$ & DMF & PMF & NMFP & PROD & $\begin{array}{c}\text { Soma de } \\
\text { postos }\end{array}$ \\
\hline - $1 \times 2$ & 616,378 & 13,450 & 4,960 & 130,822 & 22,790 & 2581,042 & 25 \\
\hline$+1 \times 3$ & 4,578 & 14,160 & 7,410 & 265,770 & 12,250 & 3253,330 & 17 \\
\hline$+1 \times 4$ & 387,266 & 16,050 & 6,610 & 220,290 & 12,580 & 2762,360 & 17 \\
\hline - $1 \times 5$ & 129,901 & 14,690 & 6,360 & 194,640 & 15,850 & 3000,520 & 20 \\
\hline - $2 \times 3$ & 555,265 & 13,340 & 5,200 & 138,700 & 21,340 & 2322,190 & 24 \\
\hline$+2 \times 4$ & 424,448 & 13,260 & 4,660 & 117,990 & 21,005 & 2472,423 & 31 \\
\hline$+2 \times 5$ & 236,967 & 12,960 & 5,060 & 117,080 & 19,330 & 2256,480 & 32 \\
\hline - $3 \times 4$ & 374,221 & 14,450 & 7,610 & 264,630 & 9,190 & 2381,250 & 18 \\
\hline$+3 \times 5$ & 104,397 & 15,300 & 7,480 & 268,920 & 10,660 & 2860,830 & 14 \\
\hline - $4 \times 5$ & 368,759 & 13,580 & 7,110 & 225,960 & 12,181 & 2360,261 & 22 \\
\hline c 'Nápoli' & & 15,810 & 7,240 & 252,350 & 9,830 & 2482,910 & \\
\hline c 'Super F100' & & 15,480 & 7,120 & 236,330 & 10,830 & 2549,160 & \\
\hline$r_{p}$ & & $-0,353$ & $-0,579$ & $-0,575$ & $0,714^{*}$ & $-0,687^{*}$ & \\
\hline$r_{s}$ & & $-0,369$ & $-0,587$ & $-0,575$ & $0,636^{*}$ & $-0,478$ & \\
\hline
\end{tabular}

$* \mathrm{P}<0,05 ;{ }^{\text {ns }}-$ não significativo.

a 1 - PI 206472; 2 - 'Long green'; 3 - 'Campineira'; 4 - 'Flórida Market'; 5 - 'E-22';

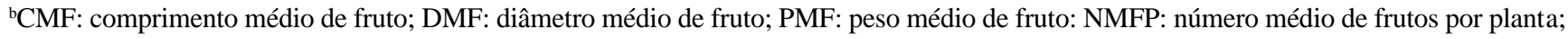
PROD: produção de frutos.

·híbrido predito, + híbrido analisado; e.

c híbrido comercial

Quadro 3 - Grupos de similaridade entre cinco acessos de berinjela estabelecidos pelo método de Tocher, a partir das distâncias generalizadas de Mahalanobis (D²). Piracicaba, USP - ESALQ, 1996.

\begin{tabular}{cl}
\hline Grupos & \multicolumn{1}{c}{ Acessos } \\
\hline I & 1 - PI 206472; 3 - 'Campineira e 5 - 'E-22' \\
II & 4 - 'Florida Market' \\
III & 2 - 'Long Green' \\
\hline
\end{tabular}

(Vicia fava L.). De acordo com estes autores, a ocorrência de associação negativa implica na existência de desvios de dominância negativos, ou seja, a presença de genes dominantes cujas expressões tendem a reduzir as estimativas do caráter. Assim, à medida que se aumenta a estimativa de $\mathrm{D}^{2}$ entre genitores, aumenta-se também a frequiência destes genes e reduz-se a expressão do caráter.

Para se estabelecer as classes de cruzamentos divergentes (CD) conforme Arunachalam \& Bandyopadhyay (1984), método 2, calculou-se a divergência genética média $(\mathrm{m})$ entre os pares de acessos, cujo valor foi $\mathrm{m}=$ 320,218 e o desvio padrão $(\mathrm{s})=198,031$ (Quadro 2). Assim, os cruzamentos fo- ram separados nas seguintes classes:

CD1 $\left(\mathrm{D}^{2} 3 \mathrm{~m}+\mathrm{s}\right): \mathrm{D}^{2} 3518,249=1 \mathrm{x}$ 2 e $2 \times 3$;

$\mathrm{CD} 2\left(\mathrm{~m}+\mathrm{s}>\mathrm{D}^{2} 3 \mathrm{~m}\right): 518,249>\mathrm{D}^{2} 3$ $320,218=1 \times 4,2 \times 4,3 \times 4$ e $4 \times 5$;

CD3 (m > D 23 m-s): $320,218>D^{2} 3$ $130,187=2 \times 5 ; \mathrm{e}$

CD4 ( $D^{2} £$ m-s): $D^{2} £ 130,187=1 x$ $3,1 \times 5,3 \times 5$.

Segundo o método 2, as classes CD2 e CD3 são as que apresentam maior freqüência de cruzamentos com bom desempenho (Arunachalan \& Bandyopadhyay, 1984). Neste estudo estas classes foram representadas pelos cruzamentos $1 \times 4,2 \times 4,3 \times 4,4 \times 5$, e $2 \times 5$, os quais ocuparam as seguintes posições na soma de postos de classificação: segunda, quarta, sexta, nona e décima respectivamente (Quadro 2).

Concluiu-se que o método 2 foi eficiente, pois, dentre cinco cruzamentos selecionados, dois produziram híbridos que ocuparam as primeiras posições na soma de postos de classificação.

Em relação ao método 3, da classificação dupla, observou-se que o acesso 1 (PI206472) apresentou bom desempenho para todos os caracteres analisados (Quadro 4), ocupando a primeira posição na soma de postos da classificação. Esse fato, segundo Lal \& Pathak (1974), evidencia alta capacidade geral de combinação, o que pode ser confirmado em Silva et al. (1999). Portanto, o acesso 1 (PI 206472) tem grande concentração de alelos favoráveis para os caracteres estudados (Vencovsky, 1970). Notou-se que o acesso 3 ('Campineira') ocupou a segunda posição e os acesso 4 ('Florida Market') e 5 ('E-22'), ocuparam a terceira posição, na soma de postos da classificação das estimativas das médias 
Quadro 4 - Médias, classificação e soma de postos da classificação dos genitores, referentes a cinco caracteres relativos à produção de frutos, em berinjela. Piracicaba, USP - ESALQ, 1996.

\begin{tabular}{lcccccccccccc}
\hline \multirow{2}{*}{ Genitor } & \multicolumn{3}{c}{ CMF $^{1}$} & \multicolumn{2}{c}{ DMF $^{2}$} & \multicolumn{2}{c}{ PMF $^{3}$} & \multicolumn{2}{c}{ NMFP $^{4}$} & \multicolumn{2}{c}{ PROD $^{5}$} & Soma de \\
\cline { 2 - 10 } & média & clas $^{*}$ & média & clas & \multicolumn{1}{c}{ média } & clas & média & clas & média & clas & postos \\
\hline $1^{\text {a }}$ & 15,633 & 2 & 6,736 & 2 & 227,420 & 2 & 13,667 & 3 & 3103,333 & 1 & 10 \\
2 & 10,540 & 5 & 3,650 & 5 & 49,776 & 5 & 32,833 & 1 & 1638,286 & 5 & 21 \\
3 & 15,800 & 1 & 6,386 & 3 & 191,750 & 3 & 13,583 & 4 & 2587,143 & 3 & 14 \\
4 & 12,263 & 4 & 9,040 & 1 & 328,380 & 1 & 5,083 & 5 & 1672,916 & 4 & 15 \\
5 & 13,116 & 3 & 5,470 & 4 & 122,133 & 4 & 21,750 & 2 & 2683,456 & 2 & 15 \\
\hline
\end{tabular}

*clas: classificação.

${ }^{1} \mathrm{CMF}$ : comprimento médio de fruto; ${ }^{2} \mathrm{DMF}$ : diâmetro médio de fruto; ${ }^{3} \mathrm{PMF}$ : peso médio de fruto; ${ }^{4} \mathrm{NMFP}$ : número médio de frutos por planta; ${ }^{5}$ PROD: produção de frutos por planta.

a 1 - PI 206472; 2 - 'Long Green '; 3 - 'Campineira'; 4 - 'Flórida Market'; 5 - 'E-22'.

(Quadro 4), sendo, portanto, selecionados para a fase seguinte do método. Por outro lado, o acesso 2 ('Long Green') obteve a quinta classificação para quatro dos cinco caracteres analisados e foi, consequentemente, descartado, pois sua soma de postos de classificação foi muito alta em relação aos demais acessos (Quadro 4).

Comparando as distâncias genéticas (Quadro 2), do acesso 1 (PI 206472), em relação aos acessos 3, 4 e 5, selecionou-se o acesso 4 ('Florida Market'), por ser o mais distante $\left(\mathrm{D}^{2}=387,226\right)$. Observou-se que o híbrido 1 x 4 (Quadro 2), ocupou a segunda melhor posição na soma de postos de classificação dentre as combinações híbridas analisadas. Assim, foi demonstrada a eficiência do método 3 em selecionar cruzamentos cujos híbridos tenham bom desempenho.

O híbrido 1 x 4 (PI206472 x 'Florida Market'), quando comparado aos híbridos 'Nápoli' e 'Super F 100' (Quadro 2), apresentou estimativas superiores para os caracteres comprimento médio de fruto $(\mathrm{CMF})$, número médio de frutos por planta (NMFP) e produção de fruto (PROD). Isto demonstra o grande potencial deste cruzamento para ser explorado comercialmente.

Apesar de os métodos utilizados serem consideradas eficientes, estes não selecionaram ou explicaram o desempenho do híbrido 3 x 5 ('Campineira' x 'E22'), que ocupou a primeira posição na soma de postos de classificação das médias (Quadro 2). Em função da estimativa de $\mathrm{D}^{2}$, entre estes acessos (Quadro 2), do desempenho de ambos os acessos
(Quadro 4) e das estimativas de capacidade geral e especifica de combinação (Silva et al., 1999), supõe-se que interações epistáticas estejam associadas ao desempenho deste híbrido. Para esclarecer esse fato, novos estudos são necessários.

\section{LITERATURA CITADA}

AMARAL JÚNIOR, A.T. Análise dialélica de betacaroteno, vitamina $C$, sólidos solúveis e produção e variabilidade em cultivares de tomateiro (Lycopersicon esculentum Mil.) via RAPD. Viçosa: UFV, 1996. 198 p. (Tese doutorado).

ARUNACHALAM, V ; BANDYOPADHYAY, A. Limits to genetic divergence for occurrence of heterosis-experimental evidence from crop plants. Indian Journal of Genetics and Plant Breeding, v. 44, n. 3, p. 548-554, 1984.

BERNARDO, R. Relationship between singlecross performance and molecular marker heterozygosity. Theoretical na Applied Genetics, v. 83, n. 5, p. 628-634, 1992.

CRUZ, C.D. Programa Genes: aplicativo computacional em genética e estatística. Viçosa: Editora UFV, 1997. 442 p.

CRUZ, C.D., CARVALHO, S.P.; VENCOVSKY, R. Estudos sobre divergência genética. I. Fatores que afetam a predição do comportamento de híbridos. Revista Ceres, Viçosa, v. 41, n. 234, p. 178-182, 1994a.

CRUZ, C.D., CARVALHO, S.P.; VENCOVSKY, R. Estudos sobre divergência genética. II. Eficiência da predição do comportamento de híbridos com base na divergência de progenitores. Revista Ceres, Viçosa, v. 41, n. 234, p. 183 190, 1994b.

DIAS, L.A.S.; KAGEYAMA, P. Multivariate genetic divergence and hybrid performance of cacao (Theobroma cacao L.). Brazilian Journal of Genetics, Ribeirão Preto, v. 20, n. 1, p. 63-70, 1997.

DIERS, B.W.; McVETTY, P.B.E.; OSBORN, T.C. Relationship between heterosis and genetic distance based on restriction fragment length polymorphism markers in Oilseed Rape (Brassica napus L.). Crop Science, v. 36, n. 1, p. 79-83, 1996.
FALCONER, D.S.; MACKAY, T.F.C. Introduction to quantitative genetics. 4. ed, Essex: Longman Group, 1996. 464 p.

FILGUEIRA, F.A.R. Manual de olericultura: cultura e comercialização de hortaliças, v. 2. São Paulo: Agronômica Ceres, 1982. 357 p.

GHADERI, A., ADAMS, M.W.; NASSIB, A.M. Relationship between genetic distance and heterosis for yield and morphological traits in dry edible bean. Crop. Science, v. 24, n. 1, p. $37-42,1984$.

GRIFFING, B. Concept of general and specific combining ability in relation to diallel crossing systems. Australian Journal of Biological Sciences, v. 9, n. 4, p. 463-493, 1956.

HALLAUER, S.R.; MIRANDA Filho, J.B. Quantitative genetics in maize breeding. 2. ed., Ames: Iowa State University Press, 1988. 468 p.

KEMPTHORNE, O.; CURNOW, R.N. The partial diallel cross. Biometrics, v. 17, n. 2, p. $229-$ 250, 1961.

LAL, S. ; PATHAK, M.M. Combining ability in brinjal. Indian Journal Genetics and Plant Breeding., v. 34, n. 3, p. 395 - 399, 1974.

MANJARREZ-SANDOVAL, P.; CARTER Jr, T.E.; WEBB, D.M.; BURTON, J.W. Heterosis in soybean and its prediction by genetic similarity measures. Crop Science, v. 37, n. 5, p. 1443-1452, 1997.

MAROOF, M.A.S.; YANG, G.P.; ZHANG, Q.; GRAVOIS, K.A. Correlation between molecular marker distance and hybrid performance in U.S. southern long grain rice. Crop Science, v. 37, n. 1, p. 145-150, 1997.

RAO, R.C. Advanced statical methods in biometrical research. New York: John Wiley and Son, 1952. 390 p.

SILVA, D.J.H.; COSTA, C.P.; CASALI, V.W.D.; DIAS, L.A.S.; CRUZ, C.D. Análise da capacidade combinatória em berinjela (Solanum melongena L.). Bragantia, Campinas, v. 58, n. 1, 1999 (no prelo).

STEEL, R.G.; TORRIE, J.H.; DICKEY, D.A. Principles and procedures of statistics: $a$ biometrical approach, 3. ed., New York: McGraw-Hill, 1997. 666 p

VALOIS, A.C.C.; SALOMÃO, A.N.; ALLEM, A.C. Glossário de recursos genéticos vegetais. Brasília: Embrapa-SPI, 1996. 62 p.

VENCOVSKY, R. Alguns aspectos teóricos e aplicados relativos a cruzamentos dialélicos de variedades. Piracicaba: ESALQ/USP, 59 p. (Tese Livre docência).

WEIR, B.S. Genetic data analysis II. Sunderland: Sinauer Associates, 1996. 445 p. 